\title{
Presentación clínica y manejo endoscópico de bola fúngica del receso del seno frontal y mucocele secundario
}

\author{
Clinical presentation and endoscopic approach of frontal sinus \\ recess fungal ball and secondary mucocele
}

José Antonio Pirrón Lozano, * Liliana Elizabeth Hernández Carpio*

\begin{abstract}
RESUMEN
La bola fúngica es el tipo más común de rinosinusitis fúngica. Se trata de una variedad de rinosinusitis no invasiva ( $\sin$ invasión tisular) y que se manifiesta típicamente en pacientes inmunocompetentes. Se le define como una aglomeración de detritos e hifas dentro de un seno paranasal. Su localización más frecuente son los senos maxilar y esfenoidal, y el agente etiológico involucrado con más frecuencia es Aspergillus $s p$. Los reportes en la literatura mundial de su presentación en el seno frontal muestran una incidencia de apenas $2 \%$ de los casos. Se realiza revisión de la literatura de las bolas fúngicas nasosinusales y del mucocele frontal tomando como referencia el caso de un paciente de 67 años de edad con antecedente de dos craneotomías quien presentó bola fúngica del receso frontal y mucocele secundario, que fue manejado quirúrgicamente mediante un abordaje endoscópico al seno frontal tipo Draf IIa.
\end{abstract}

Palabras clave: Bola fúngica frontal, mucocele frontal, Draf.

Nivel de evidencia: IV

\section{INTRODUCCIÓN}

La bola fúngica (mal llamada micetoma o aspergiloma) es una forma de rinosinusitis fúngica no invasiva, la cual se caracteriza por ausencia de invasión tisular típicamente en pacientes inmunocompetentes. ${ }^{1}$ Algunos estudios reportan cierto grado de inmuno-

\begin{abstract}
Fungal ball is the most common type of fungal rhinosinusitis. It is a variety of non-invasive rhinosinusitis (without tissue invasion) that typically occurs in immunocompetent patients. It is defined as an agglomeration of detrites and hyphaes within a paranasal sinus. Its most common location is the maxillary and sphenoidal sinuses, and the most frequently involved etiological agent is Aspergillus sp. Reports in the world literature of its presentation within the frontal sinus show an incidence of only $2 \%$ of the cases. A review of the literature on nasosinusal fungal balls and frontal mucoceles was made, taking as a reference the case of a 67-year-old male patient with a history of two craniotomies who presented with fungal ball in the frontal sinus recess and secondary mucocele that was managed by an endoscopic Draf IIa approach to the frontal sinus.
\end{abstract}

Keywords: Frontal fungal ball, frontal mucocele, Draf.

Level of evidence: $I V$

compromiso (ej. incidencia de hasta $4 \%$ de diabetes en pacientes con bola fúngica). ${ }^{2}$ Los hongos involucrados son: Aspergillus (A. fumigatus y A. flavus), Alternaria, Pseudallescheria, Mucor, Bipolaris y Rhizopus. Sólo $23-50 \%$ de los cultivos son positivos. Es una aglomeración no invasiva de detritos e hifas dentro de algún seno paranasal, siendo el seno maxilar el más

\section{* Otorrinolaringólogo, Miembro del Cuerpo Médico del Centro Médico ABC.}

Recibido para publicación: 10/06/2019. Aceptado: 28/11/2019.

Este artículo puede ser consultado en versión completa en: www.medigraphic.com/analesmedicos
Correspondencia: José Antonio Pirrón Lozano Av. Vasco de Quiroga Núm. 4299, consultorio 904, Col. Santa Fe, 05348,

Alcaldía Cuajimalpa de Morelos, CDMX. E-mail: joseantoniopirron@ hotmail.com 
afectado (75\%) seguido del seno esfenoidal. El seno frontal se ve afectado en $2 \%$ de los casos. Su presentación es inespecífica o asintomática (puede llevar a un diagnóstico tardío), aunque pudiera manifestarse como una sinusitis crónica unilateral recalcitrante con rinorrea, obstrucción nasal y algias craneofaciales; otros síntomas incluyen: epistaxis, cacosmia, tos, disfonía, rinorrea posterior, e incluso proptosis y síntomas neurológicos como convulsiones y alteraciones visuales cuando se afecta el seno esfenoidal. La edad media de presentación es de 64 años con relación de 2:1 a favor de las mujeres. Su diagnóstico se basa en la endoscopia nasal y estudios de imagen (tomografía), y se confirma mediante estudio histopatológico. El tratamiento es esencialmente quirúrgico. ${ }^{1,3,4}$

Los mucoceles son sacos epiteliales de contenido mucoso por obstrucción de algún seno paranasal (ej. por pólipos o tumores), $90 \%$ se desarrollan en el seno frontal. No hay prevalencia de género y son más comunes entre los 40 y 60 años de edad, se manifiesta con dolor/presión facial-frontal, edema periorbitario, proptosis, celulitis periorbitaria, diplopía, rinorrea y obstrucción nasal. ${ }^{5,6}$ Los factores predisponentes son: traumatismos y cirugías regionales previas. Pueden afectar la órbita y elementos intracraneales por su efecto expansivo-destructivo. La tomografía y resonancia confirman el diagnóstico. El abordaje endoscópico al seno frontal (Draf) es el tratamiento de elección. Casos más complejos pueden requerir de abordajes abiertos o combinados. ${ }^{5-7}$

\section{PRESENTACIÓN DEL CASO}

Paciente masculino de 67 años de edad, con el antecedente quirúrgico de dos craneotomías realizadas hace 20 y 25 años respectivamente. En la primera ocasión, dicho procedimiento fue secundario a un traumatismo craneoencefálico por el cual fue sometido a un drenaje de hematoma subdural. La segunda craneotomía fue debida al clipaje de un aneurisma cerebral. Cuenta también con dos antecedentes adicionales de importancia: cirugía nasal funcional previa hace 10 años (septumplastia), y síntomas clínicos recurrentes de rinitis alérgica, los cuales controla sólo de manera esporádica con antihistamínicos, no se realizaron previamente estudios específicos para el diagnóstico de alergias.

Acudió a la consulta de otorrinolaringología por un cuadro clínico de tres meses de evolución caracterizado por dolor facial de intensidad nueve de 10 , de inicio súbito, recurrente y de localización de predominio maxilar y orbitario izquierdos. Otros síntomas asociados con su cuadro: lagrimeo izquierdo intermi- tente, rinorrea blanquecina anterior y posterior, ligera obstrucción nasal de predominio izquierdo y otalgia ocasional del oído izquierdo. A la exploración física el paciente se encontraba afebril y con presencia de edema palpebral izquierdo, pero con movimientos oculares, agudeza visual y reflejos oculares conservados. Evidenciaba rinorrea blanquecina espesa por el meato medio izquierdo y edema e hiperemia de la mucosa a la endoscopia nasal en consultorio.

Ante la sospecha de una complicación orbitaria por rinosinusitis agudizada (probable celulitis preseptal izquierda) se realizó un estudio tomográfico inicial urgente simple de senos paranasales (Figuras 1 y 2), en el cual se observó la presencia de una imagen de aspecto heterogéneo que ocupaba y ampliaba el área del receso del seno frontal izquierdo y celdillas etmoidales anteriores adyacentes a la lámina papirácea ipsilateral, así como el seno frontal izquierdo totalmente ocupado por una densidad de aspecto homogéneo; se apreció también un ligero desplazamiento inferolateral del globo ocular, pero sin datos de formación de absceso subperióstico. De igual forma, se visualizó una zona de pérdida de continuidad ósea en la tabla anterior del seno frontal izquierdo producto de las craneotomías y traumatismo craneoencefálico previos. La imagen de resonancia magnética (Figura 3) mostró datos de mucocele del seno frontal izquierdo, se apreció una imagen hipointensa/isointensa con reforzamiento periférico en seno frontal izquierdo en fase T1,

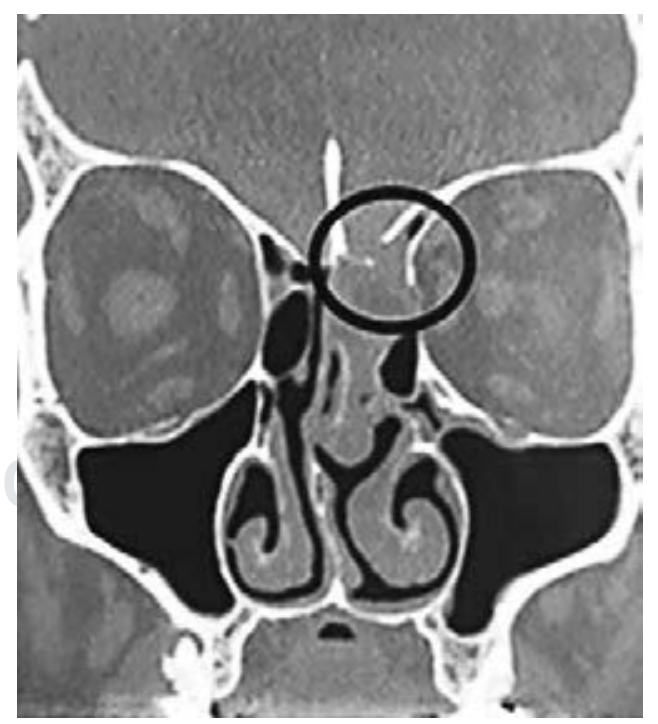

Figura 1: Tomografía simple de senos paranasales, corte coronal. Receso frontal izquierdo ampliado y ocupado por una imagen heterogénea que desplaza al septum a la derecha, así como dehiscencias parciales en lámina papirácea y fóvea etmoidal. 


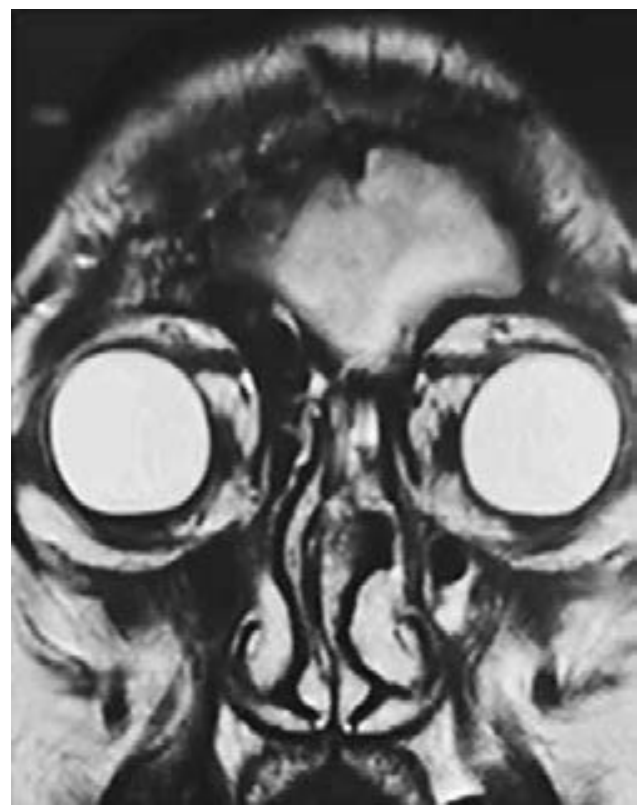

Figura 2: Estudio de resonancia magnética fase T2, corte coronal. Contenido hiperintenso en seno frontal y lesión de densidades mixtas a nivel del receso del seno frontal ipsilateral. Obsérvese el desplazamiento lateral del globo ocular izquierdo.

e hiperintensa en T2. En ambas fases se observaron densidades mixtas (hipotensas e hiperintensas) a nivel del receso del seno frontal izquierdo, el cual se aprecia ampliado con relación al lado derecho.

El paciente recibió manejo médico inmediato con levofloxacino, esteroides nasales y sistémicos, irrigaciones nasales con solución fisiológica, descongestionante tópico nasal (oximetazolina) y manejo del dolor con tramadol y ketorolaco, con lo cual remitió el edema palpebral y el dolor disminuyó de manera significativa. Posteriormente fue programado para manejo quirúrgico.

Durante la cirugía se observó la presencia de material de aspecto micótico de consistencia pastosa/ endurecida y coloración blanca amarillenta caseosa que ocupaba la zona del receso del seno frontal izquierdo, el cual al ser retirado produjo el drenaje de rinorrea purulenta blanquecina espesa que ocupaba el interior del seno frontal izquierdo. En el reporte del estudio histopatológico de las lesiones micóticas con tinciones de PAS y Grocott resultó negativo, y también se observó una colonización bacteriana multifocal con microorganismos cocoides Gram positivos, así como una inflamación aguda inespecífica. El cultivo de la secreción proveniente del seno frontal fue positivo para Staphylococcus aureus.
La evolución del paciente a un año y medio de la cirugía ha sido satisfactoria.

Actualmente recibe el control de su atopia mediante inmunoterapia subcutánea.

\section{DISCUSIÓN}

La relevancia de presentar este caso clínico en particular radica en que el médico pueda sospechar la existencia de una bola fúngica en un paciente que manifieste rinosinusitis unilateral recalcitrante a tratamiento médico asociada con algias craneofaciales, así como tener las herramientas para su diagnóstico y manejo quirúrgico adecuados. De igual manera, se pretende hacer hincapié en interrogar los antecedentes de cirugías o traumatismos craneofaciales en pacientes con sinusitis complicadas por la probabilidad de presencia de mucoceles y guiar al médico en el manejo de estas lesiones.

Los criterios diagnósticos clínico-patológicos para la bola fúngica de senos paranasales se sintetizan a continuación: ${ }^{8}$

1. Evidencia radiológica (tomografía) de opacificación de un seno paranasal con o sin microcalcificaciones asociadas.

2. Material mucopurulento, arcilloso o caseoso en el interior del seno afectado.

3. Aglomeración de hifas separada, pero adyacente a la mucosa sinusal.

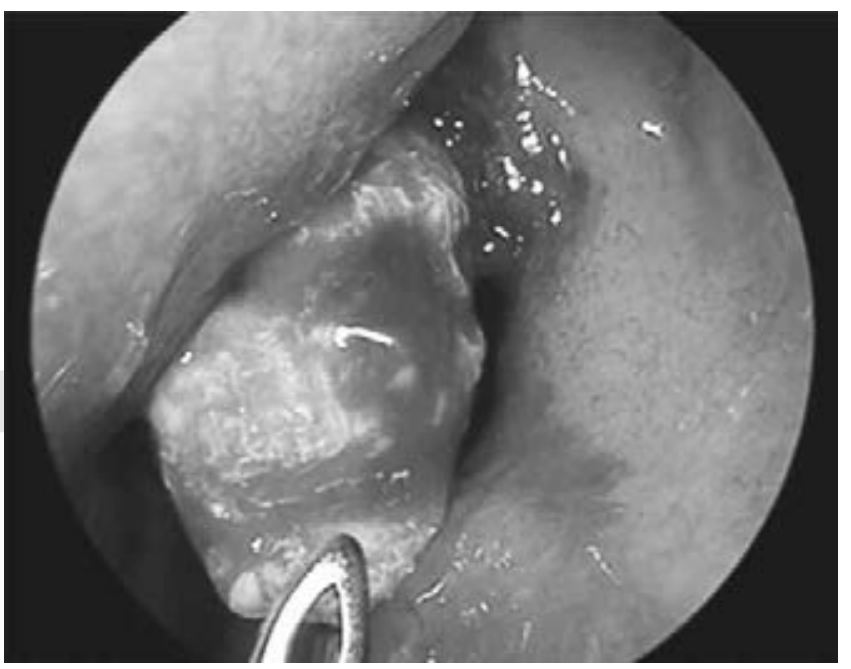

Figura 3: Imagen endoscópica transoperatoria. Extracción de fragmentos de material de aspecto micótico/caseoso provenientes del área del receso del seno frontal izquierdo, con reporte negativo en cultivo para hongos. 
4. Inflamación crónica de intensidad variable en la mucosa adyacente a la bola fúngica (linfocitos, células plasmáticas, eosinófilos). No debe detectarse mucina alérgica en la tinción con hematoxilina-eosina. Sin predominio de eosinófilos ni granulomas.

5. Ausencia de invasión de la mucosa, de los vasos sanguíneos o del hueso subyacente en el estudio histológico con tinción de Gomori-plata u otras tinciones especiales para hongos.

El primer caso de bola fúngica en senos paranasales reportado en la literatura fue realizado por Mackenzie en el año de 1894. El término aspergiloma se empleaba más comúnmente antes de conocerse que esta identidad también podría ser provocada por otros hongos como Mucor, Alternaria y Bipolaris.

La fisiopatología de la bola fúngica sigue siendo controvertida. La teoría aerogénica de Milosev descrita desde 1969 sugiere que las esporas de los hongos, las cuales se encuentran en abundancia y de manera normal en el medio ambiente, se depositan en la mucosa nasosinusal por un mecanismo de inhalación directa, y adquieren capacidad patogénica cuando se crean condiciones anaeróbicas en el interior del seno afectado. Otros autores como Stammberger han propuesto que el exudado purulento encontrado en los periodos de agudización de una rinosinusitis crónica puede predisponer o representar un medio de crecimiento idóneo para el crecimiento de los hongos. Otra de las teorías acerca de la patogénesis de las bolas fúngicas es la teoría odontogénica o «iatrogénica», existiendo el antecedente de procedimientos dentales previos (ej. endodoncias con empleo de sellantes del canal dental que contienen óxido de zinc, el cual es capaz de activar el metabolismo del Aspergillus) hasta en $85 \%$ de los casos se presume pudo existir una comunicación oroantral accidental; sin embargo, dicho antecedente sólo es de relevancia en el caso de la bola fúngica maxilar. Algunos de los factores predisponentes generales para bola fúngica descritos en la literatura son la diabetes, residencia en áreas rurales, cirugías nasosinusales previas, variantes anatómicas nasosinusales (ej. desviaciones septales o cornetes paradójicos en $15 \%$ ), y procedimientos dentales previos sobre el maxilar superior ipsilateral. Se ha sugerido que la baja incidencia de bolas fúngicas del seno frontal se debe principalmente a la complejidad anatómica del receso del seno frontal y a la localización anterosuperior del seno que lo hace menos accesible a las esporas inhaladas. Bernardini y colaboradores reportaron en 2007 algunos casos similares a los de nuestro caso, ${ }^{9} \mathrm{y}$ sugieren que para que una bola fúngica frontal se de- sarrolle deben coexistir dos condiciones de manera simultánea: inhalación de esporas hacia el seno frontal, y obstrucción del ostium del seno frontal. La atopia no es más frecuente en los pacientes con bola fúngica que en la población general.

Las calcificaciones hiperdensas encontradas hasta en $75 \%$ de los estudios tomográficos se deben a los niveles elevados de zinc, calcio, hierro y manganeso de las hifas micóticas. Sólo en los casos de sospecha de complicaciones graves por rinosinusitis secundaria o de patología tumoral está indicado un estudio complementario de resonancia magnética.

El diagnóstico definitivo es esencialmente histopatológico, en el que se observan conglomerados de hifas sin evidencia de lesión tisular ni de tejido de granulación, con zonas densas de crecimiento alternadas con otras de menor densidad dando una apariencia concéntrica de «piel de cebolla».

Los cultivos suelen ser negativos; cuando son positivos, hasta $93 \%$ de los casos reportan Aspergillus fumigatus. . $^{1,3}$

El tratamiento de la bola fúngica consiste en la resección quirúrgica completa, con lo cual se describe una tasa de recurrencia menor de 5\%. Los fármacos antimicóticos no están indicados en esta entidad, los esteroides nasales pueden tener un papel para el manejo de la inflamación de la mucosa nasosinusal en el postoperatorio. Las complicaciones reportadas por no tratar las bolas fúngicas de los senos paranasales son: rinosinusitis bacteriana recurrente, mucoceles, pioceles, y complicaciones neurológicas como neuritis óptica, oftalmoplejía y convulsiones. El manejo quirúrgico de elección para la resección de las bolas fúngicas nasosinusales es mediante las técnicas endoscópicas. Específicamente para los casos de afección del seno frontal, los procedimientos Draf IIa, IIb y III (Lothrop modificado) son los más empleados; ${ }^{6,9,10}$ sin embargo, en los casos en los cuales no se logre una visualización y exposición suficiente de la cavidad del seno frontal, podrían estar indicados los abordajes externos como la cirugía osteoplástica del seno frontal o abordajes combinados. Los abordajes endoscópicos tipo Draf IIb y III se emplean más comúnmente en casos complejos y/o con hiperneumatización del seno frontal donde se dificulta el acceso hacia las regiones laterales y anteriores del seno. En manos expertas, estos dos procedimientos endoscópicos son una alternativa válida a los abordajes externos clásicos para el seno frontal. En el caso del paciente del presente artículo se utilizó un abordaje endoscópico tipo Draf IIa, ya que el seno frontal derecho se encontraba hipoplásico o casi ausente, y la bola fúngica en el receso del seno frontal izquierdo y 
el mucocele frontal izquierdo produjeron un efecto expansivo o de ampliación que facilitó el drenaje y produjo cavidades suficientemente abiertas que permitían un drenaje y ventilación adecuados. El propósito de la cirugía es la restauración de la ventilación y drenaje fisiológicos del seno afectado, así como permitir el acceso de medicamentos tópicos en caso necesario. . $^{3,7,9}$

La presentación del mucocele frontal en este paciente tuvo como principal factor predisponente el traumatismo craneal y la craneotomía realizada para el drenaje del hematoma subdural; sin embargo, se deben considerar dos factores adicionales: la cirugía nasal previa que generó sinequias obstructivas en la zona del meato medio, y la bola fúngica formada en la región del receso del seno frontal. En este caso sólo se encontró una dehiscencia parcial del tercio inferior de la lámina papirácea izquierda y no existieron erosiones hacia el techo orbitario. Es de suma relevancia que en estos casos se realice una valoración oftalmológica completa y que todos aquellos pacientes que se presenten con una ptosis palpebral dolorosa (o parálisis dolorosa del tercer nervio craneal), con o sin afección pupilar, sean sometidos a estudios de imagen craneal y orbitaria de manera urgente. Los mucoceles deben formar parte de la lista de los diagnósticos diferenciales en un paciente que padezca ptosis palpebral. El tratamiento definitivo, al igual que en el caso de la bola fúngica, es quirúrgico, y la cirugía endoscópica es el procedimiento de elección. Aquellos casos más complejos requerirán cirugías más radicales o abordajes abiertos. ${ }^{5-7}$ La cirugía endoscópica mínima en estos pacientes se ha descrito mediante una sinusotomía frontal tipo Draf IIa, aunque en los casos en los que exista un diámetro anteroposterior estrecho del ostium del seno frontal o en donde el engrosamiento óseo sea considerable, se sugieren abordajes tipo Draf IIb o Draf III. ${ }^{10}$

\section{CONCLUSIONES}

Debe sospecharse de una bola fúngica sinusal en pacientes que se presenten con un cuadro clínico de rinosinusitis crónica recalcitrante unilateral. El diagnóstico se basa en los hallazgos clínicos, de imagen (calcificaciones hiperdensas sinusales en la tomografía) y transoperatorias (material de aspecto caseoso o arcilloso) y se confirma mediante el estudio histopatológico.

El tratamiento de elección para la bola fúngica y los mucoceles es eminentemente quirúrgico, siendo de elección los abordajes endoscópicos al seno frontal tipo Draf IIa, IIb y III. La elección entre estos procedimientos normalmente se basa dependiendo de la severidad y extensión de la patología, pueden llegar a ser necesarios los abordajes abiertos o combinados en los casos más complejos.

\section{BIBLIOGRAFÍA}

1. Lop-Gros J, Gras-Cabrerizo JR, Bothe-González C, Montserrat-Gili J, Sumarroca-Trouboul A, Massegur-Solench $\mathrm{H}$. Bola fúngica sinusal: análisis de nuestra casuística. Acta Otorrinolaringol Esp. 2016; 67 (4): 220-225.

2. Robey A, O'Brien E, Richardson B, Baker J, Poage D, Leopold D. The changing face of paranasal sinus fungus balls. Ann Otol Rhinol Laryngol. 2009; 118 (7): 500-505.

3. Grosjean P, Weber R. Fungus balls of the paranasal sinuses: a review. Eur Arch Otorhinolaryngol. 2007; 264: 461-470.

4. Bosi GR, de Braga GL, de Almeida TS, de Carli A. Fungus ball of the paranasal sinuses: Report of two cases and literature review. Int Arch Otorhinolaryngol. 2012; 16 (2): 286-290.

5. Kennedy A, Chowdhury H, Athwal S, Baddeley P. Frontal sinus mucocoele: a rare cause of ptosis. BMJ Case Rep. 2015; 2015. pii: bcr2015211068.

6. Sama A, McClelland L, Constable J. Frontal sinus mucocoeles: new algorithm for surgical management. Rhinology. 2014; 52 (3): 267-275.

7. Aguilar SJA, Pirrón LJA. Abordaje osteoplástico para el tratamiento de mucopiocele frontal. Comunicación de un caso. An Orl Mex. 2009; 54 (1): 40-44.

8. deShazo RD, O'Brien M, Chapin K, Soto-Aguilar M, Swain R, Lyons $\mathrm{M}$ et al. Criteria for the diagnosis of sinus mycetoma. J Allergy Clin Immunol. 1997; 99 (4): 475-485.

9. Bernardini E, Karligkiotis A, Fortunato S, Castelnuovo P, Dallan I. Surgical and pathogenic considerations on frontal fungus ball. Eur Arch Otorhinolaryngol. 2017; 274: 24932497.

10. Nomura K, Hidaka H, Arakawa K, Sugawara M, Ozawa D, Okumura Y et al. Outcomes of frontal mucoceles treated with conventional endoscopic sinus surgery. Acta Otolaryngol. 2015; 135 (8): 819-823. 